\title{
Histoid leprosy: de novo case with atypical presentation
}

\author{
SOUMYA AGARWAL ${ }^{\mathrm{a}}$, TITIKSHA HANS ${ }^{\mathrm{a}}$, \\ SHIVANI YADAV ${ }^{\mathrm{a}}$ \& ANSHUL CHOUDHARY ${ }^{\mathrm{a}}$ \\ a Department of Dermatology, Era's Lucknow Medical College and hospital, \\ Uttar Pradesh, India
}

\begin{abstract}
Accepted for publication 16 April 2019
Summary Histoid leprosy is a rare kind of lepromatous leprosy with specific clinicopathologic manifestations.It usually follows treatment failure especially after dapsone monotherapy. Occasionally it occurs de novo without any history of inadequate or irregular treatment. We hereby report a case of de novo histoid leprosy in a 17 year old boy who presented with atypical features, including involvement of flexures, hypopigmented macules topped with nodules, and de novo occurrence.
\end{abstract}

Keywords: Histoid Hansen's, de novo histoid leprosy

\section{Introduction}

Histoid leprosy is an uncommon variant of lepromatous leprosy presenting as cutaneous or subcutaneous nodular or plaque-like lesions arising from apparently normal skin; it may affect the face, back, limbs and the skin over bony prominences and in very severe cases mucous membranes may be affected. ${ }^{1}$ It is characterised by specific histopathological and unique bacterial morphology. We report here a case of de novo histoid leprosy in a 17 year old boy presenting with multiple nodules, yellowish plaques and hypopigmented macules.

\section{Case report}

A 17 year old boy presented to us with multiple hypopigmented skin lesions and skin coloured nodules over the face, trunk and extremities for the past 5 years. The lesions first appeared over the left ankle and gradually progressed to the dorsum of left foot, both knees, upper limbs, back and around the eyes. There was no history of loss of sensation over the lesions, nasal stuffiness, swelling of feet, sudden exacerbations of the lesions or neuritic pain. The patient denied any drug intake, fever, myalgia, spontaneous blistering/ulceration, testicular pain, and ocular complaints. There was no family history of Hansen's disease or any history of previous treatment. Cutaneous examination revealed the presence of multiple, well defined, discrete to coalescent, firm, non-tender, skin coloured shiny nodules over the back, axillae, knees 


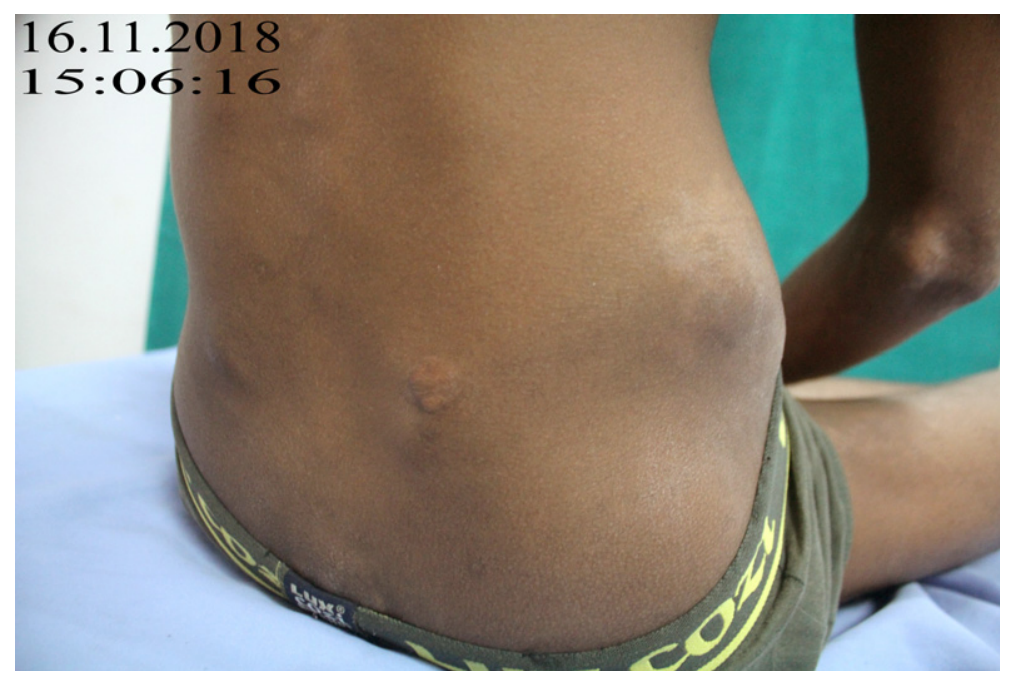

Figure 1. Skin coloured nodule over the back.

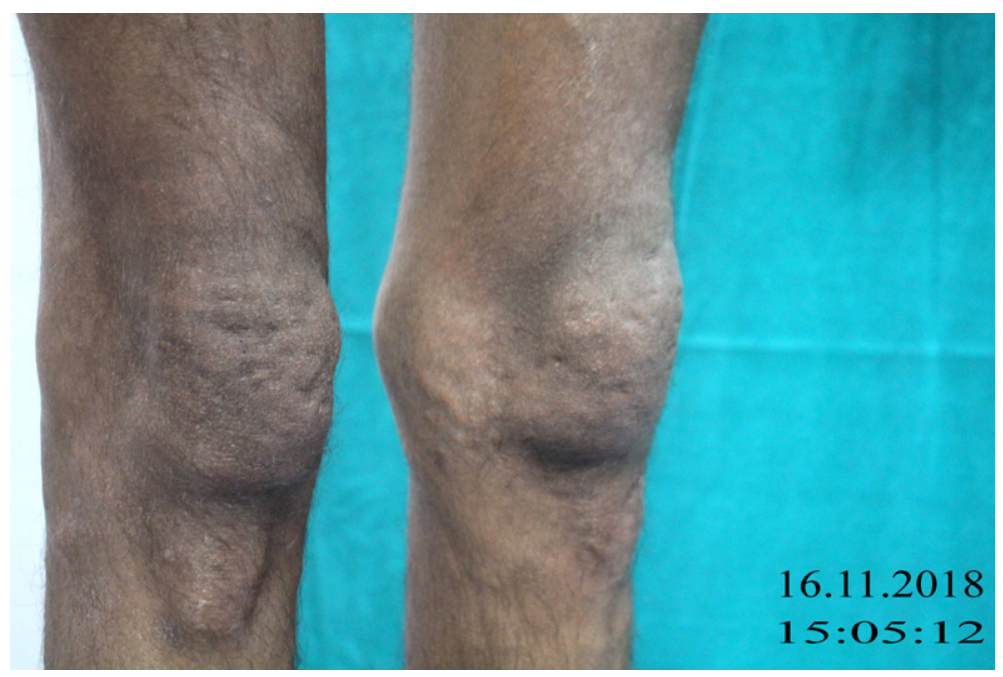

Figure 2. Hypopigmented yellowish plaques and nodules over the knees and thighs.

and thighs (Figures 1,2). There were multiple hypopigmented macules of varying size, from $0.5 \times 0.5 \mathrm{~cm}$ to $2 \times 2 \mathrm{~cm}$ over the back (Figures 3,4 ) and yellow, well-defined, discrete plaques over the medial canthi of eyes and eyelids (Figure 5). The extensor surfaces of the elbows showed atrophic scars (Figure 6).

Neurological examination revealed bilaterally symmetrical thickening of ulnar nerves, common peroneal nerves and radial cutaneous nerves. However, pain, touch and temperature sensation over the lesions were intact. The rest of the musculoskeletal and neurological examination was normal. There was no glove and stocking anaesthesia. Based on the history and examination, a differential diagnosis of histoid leprosy, post kala azar dermal leishmaniasis 


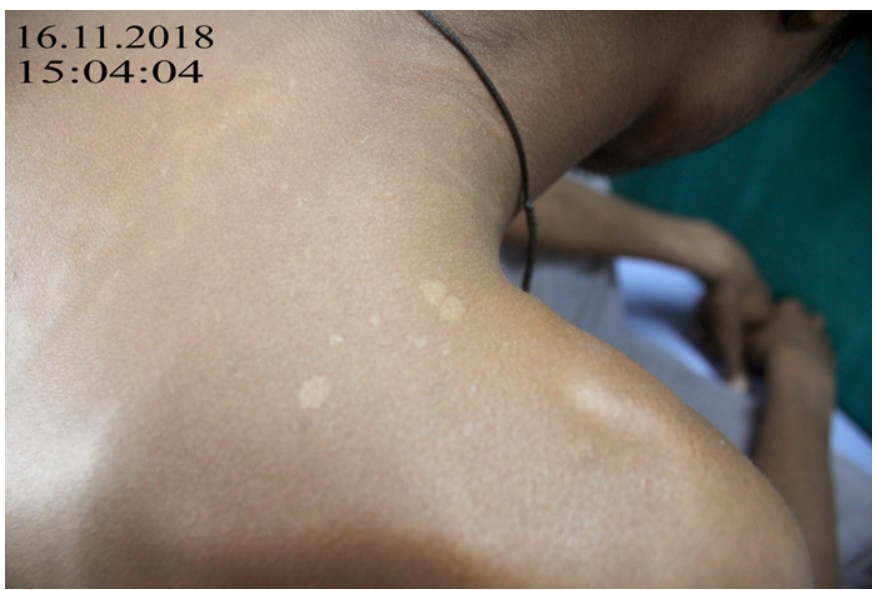

Figure 3. Hypopigmented macules over the shoulder region.

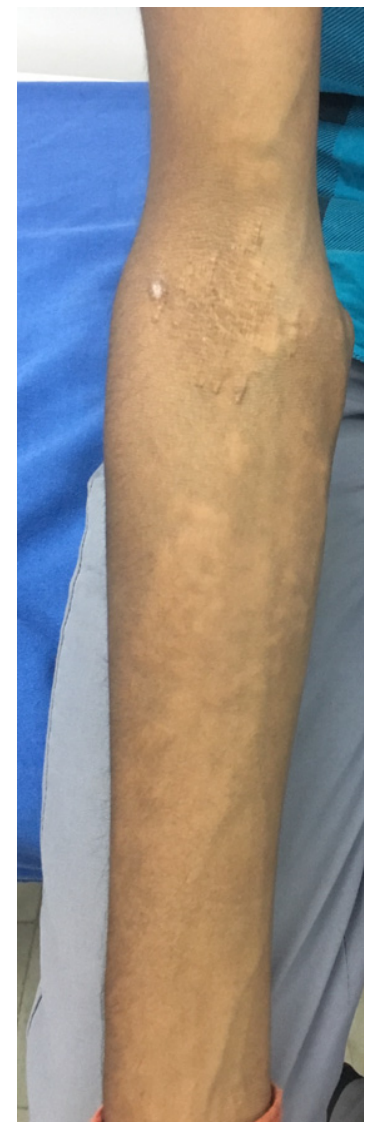

Figure 4. Macular and papular lesions over the right arm and cubital fossa. 


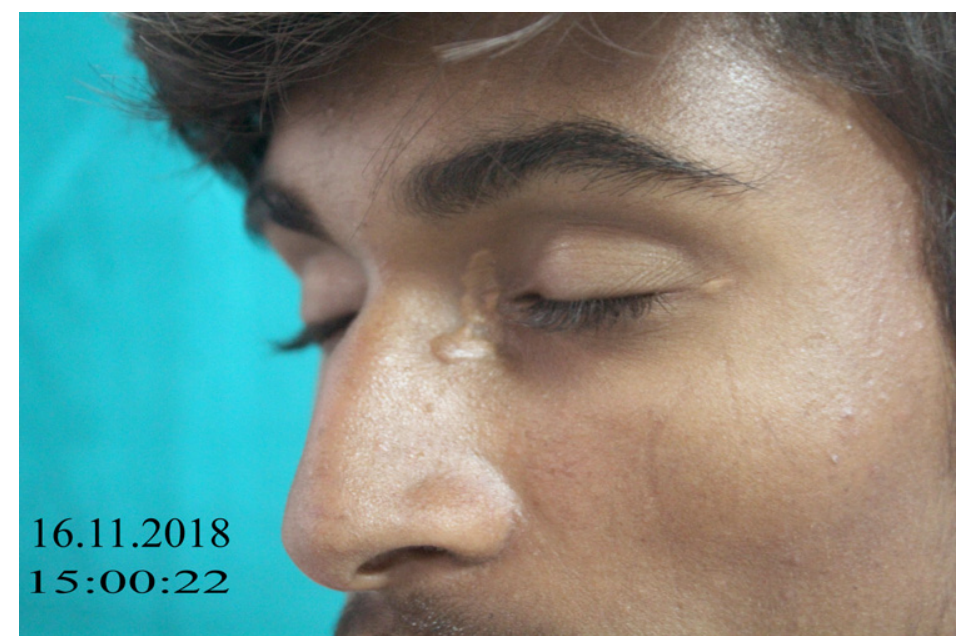

Figure 5. Yellowish plaques over medial canthi of eyes.

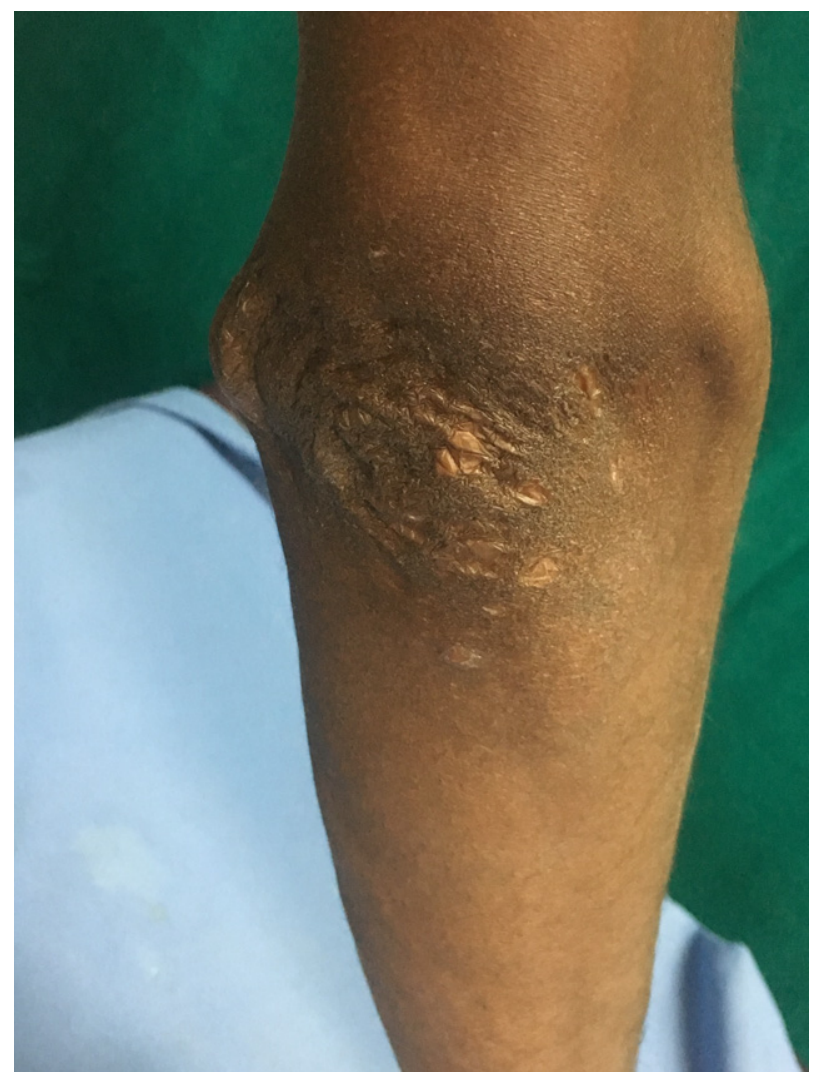

Figure 6. Atrophic scars over the elbows. 


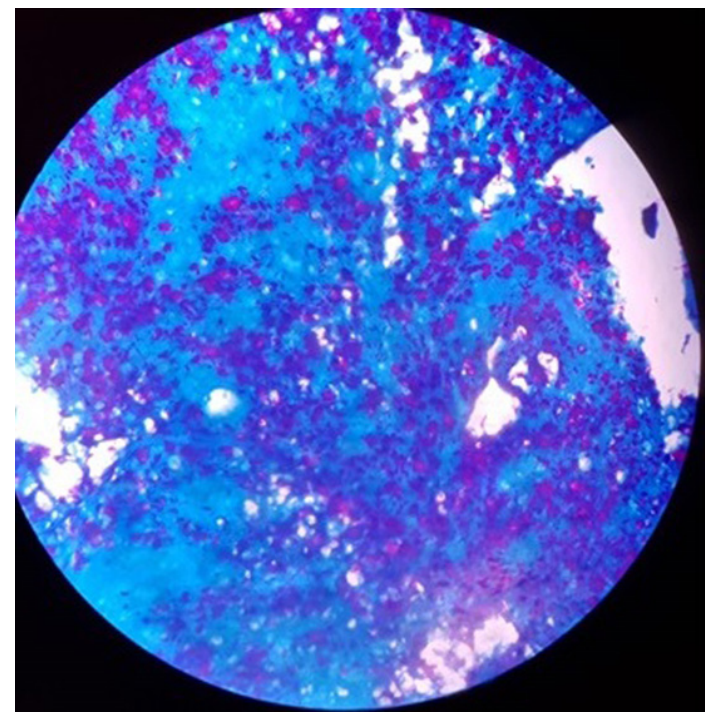

Figure 7. Photomicrograph showing acid fast bacilli in singles and in clumps (Ziehl-Neelsen stain, 100X).

(PKDL), and sarcoidosis was considered. The routine investigations, including complete blood count, liver and renal function tests, blood sugar levels and urine examination, were within normal limits. The slit skin smear examination from the right ear lobe and face was positive for acid-fast bacilli (Bacteriological Index 5+; Figure 7). Histopathological examination of the nodules revealed spindle shaped histiocytes arranged in a storiform pattern along with lymphocytes arranged in sheets as well as around adnexal structures (Figure 8). The diagnosis of histoid leprosy was confirmed and the patient was started on rifampicin $600 \mathrm{mg}$ once a month, clofazimine $300 \mathrm{mg}$ once a month and $50 \mathrm{mg}$ on alternate days, and Dapsone $100 \mathrm{mg}$ daily. The nodular lesions showed partial resolution after 3 months of treatment.

\section{Discussion}

Histoid leprosy was originally described by Wade in 1960 and is regarded as a rare type of lepromatous leprosy which has a prevalence of around 3\% among Indian leprosy patients, usually occurring after inadequate or irregular treatment of leprosy. ${ }^{1,2}$ It may occasionally present de novo without any history of treatment. ${ }^{2}$

Clinically, histoid lesions usually appear as smooth, shiny, hemispherical, dome shaped, non-tender soft to firm nodules which may be superficial, subcutaneous or fixed deeply under the skin and plaques or pads appearing on otherwise normal looking skin. ${ }^{3,4}$ They usually occur on extensor surface of the extremities, back and buttocks. They may be clustered on the face or localised to the bony prominences over the knees and elbows. ${ }^{1,5}$ Mucosa and genitalia may be involved in severe cases.

The notable histopathological findings include epidermal atrophy as a result of the underlying leproma and grenz zone located immediately below the epidermis. The lesion consist of fusiform histiocytes arranged in a whorled, criss-cross or storiform pattern. ${ }^{3,6}$ The histiocytes contain numerous bacilli and slightly vacuolated cytoplasm. The bacilli are intact and rodshaped in contrast to the fragmented forms seen in lepromatous leprosy. ${ }^{7}$ 


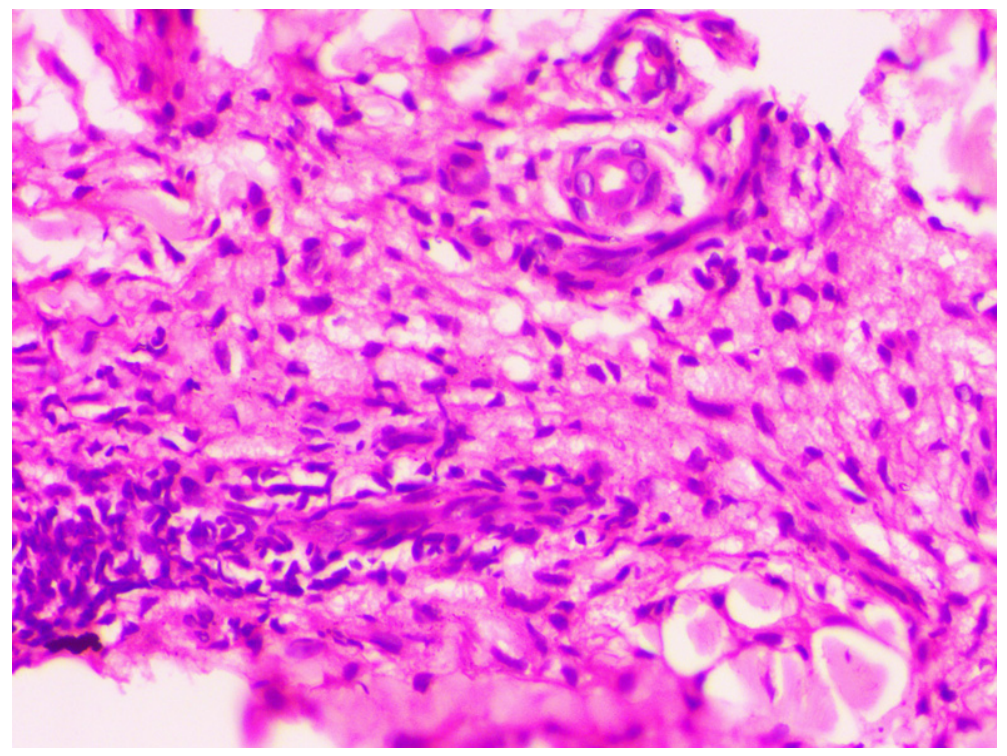

Figure 8. Spindle shaped histiocytes and lymphocytes in storiform pattern (Haematoxylin and Eosin stain 40X).

In our case the clinical picture was confirmed by slit skin smear and histopathological examination.

We report this case owing to its rarity and atypical presentation with involvement of flexures, the presence of hypopigmented macules along with nodules, and its de novo occurrence (without any history of previous treatment).

It is crucial for all dermatologists to be aware of such unusual manifestations and the management of histoid leprosy; a high index of clinical suspicion is required to diagnose such cases. Early diagnosis and management of histoid leprosy is important as these patients have a high bacillary load, might act as a reservoir of leprosy and continue to transmit the disease for a long duration.

\section{References}

1 Bhat YJ, Hassan I, Yaseen A, Wani R. De novo histoid leprosy: a case report from a post elimination area. Indian J Dermatol, 2015; 60: 214.

2 Ebrahimi A, Ramazani M, Faramarzi A, Seidmohammaddoulabi S, Kavoussi H. Case report of a histoid leprosy patient. J Pak Assoc Dermatol, 2013; 23(3): 341-344.

3 Sehgal VN, Srivastava G. Histoid leprosy. A review. Int J Dermatol, 1985; 24: 286-292.

4 Kaur I, Dogra S, De D, Saika UN. Histoid leprosy: a retrospective study of 40 cases from India. Br J Dermatol, 2009; 160: 305-310.

5 Annigeri SR, Metgud SC, Patel JR. Lepromatous Leprosy of histoid type: A case report. Indian J Med Microbiol, 2207; 25: 70-71.

6 Murthy SV, Rao SM, Thejasvini Mannan K. De novo Histoid Leprosy. J Lab Physicians, 2011; 3: 110-112.

7 Mansfield RC. Histoid leprosy. Arch Pathol, 1969; 87: 580-585. 